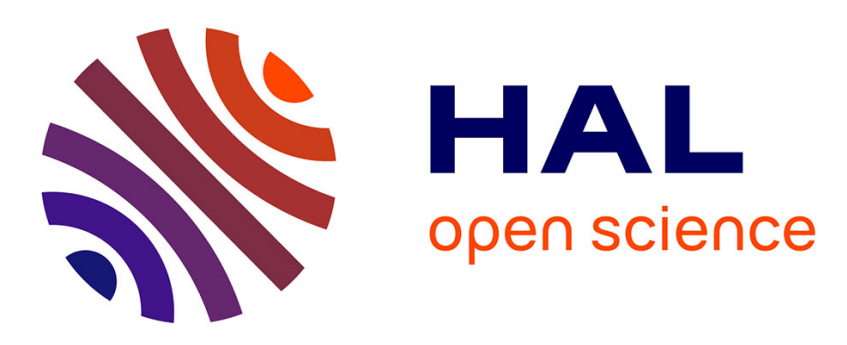

\title{
On the gas phase fragmentation of protonated uracil: a statistical perspective
}

\author{
Estefania Rossich-Molina, Jean-Yves Salpin, Riccardo Spezia, Emilio
}

Martinez-Nunez

\section{To cite this version:}

Estefania Rossich-Molina, Jean-Yves Salpin, Riccardo Spezia, Emilio Martinez-Nunez. On the gas phase fragmentation of protonated uracil: a statistical perspective. Physical Chemistry Chemical Physics, 2016, 18 (22), pp.14980-14990. 10.1039/c6cp01657j . hal-01386210

\section{HAL Id: hal-01386210 https://hal.science/hal-01386210}

Submitted on 13 Feb 2019

HAL is a multi-disciplinary open access archive for the deposit and dissemination of scientific research documents, whether they are published or not. The documents may come from teaching and research institutions in France or abroad, or from public or private research centers.
L'archive ouverte pluridisciplinaire HAL, est destinée au dépôt et à la diffusion de documents scientifiques de niveau recherche, publiés ou non, émanant des établissements d'enseignement et de recherche français ou étrangers, des laboratoires publics ou privés. 


\title{
On the gas phase fragmentation of protonated uracil: a statistical perspective
}

\author{
Estefanía Rossich Molina, ${ }^{\text {a,b }}$ Jean-Yves Salpin, ${ }^{\text {a,b }}$ Riccardo Spezia ${ }^{a, b *}$ and Emilio \\ Martínez-Núñez ${ }^{c *}$
}

The potential energy surface of protonated uracil has been explored with an automated transition state search procedure, resulting in the finding of 1398 stationary points and 751 reactive channels, which can be categorized into isomerizations between pairs of isomers, unimolecular fragmentations and bimolecular reactions. The use of statistical RiceRamsperger-Kassel-Marcus (RRKM) theory and Kinetic Monte Carlo (KMC) simulations allowed us determining the relative abundances of each fragmentation channel as a function of the ion's internal energy. The KMC/RRKM product abundances are compared with novel mass spectrometry (MS) experiments in the collision energy range 1-6 eV. To facilitate the comparison between theory and experiments, further dynamics simulations are carried out to determine the fraction of collision energy converted into the ion's internal energy. The KMC simulations show that the major fragmentation channels are isocyanic acid and ammonia losses, in good agreement with experiments. The third predominant channel is water loss according to both theory and experiments, although the abundance obtained in the KMC simulations is very low, suggesting that non-statistical dynamics might play an important role for this channel. Isocyanic acid $\left(\mathrm{HNCOH}^{+}\right)$is also an important product in the $\mathrm{KMC}$ simulations, although its abundance is only significant at internal energies not accessible in the MS experiments.

\section{Introduction}

Unravelling the fragmentation mechanisms observed in tandem mass spectrometry (MS/MS) or collision induced dissociation (CID) is a complicated task. Computational chemistry is a useful tool, and two main approaches are generally employed: (i) mapping the potential energy surface (PES) connecting the reactant with the product ions; (ii) using chemical dynamics of collisions. In the first case, one has to carefully identify all the minima and transition states (TSs) connecting the reactants with the products. This can be done either using chemical intuition, or relying on automated methods, i.e., with little human intervention. The latter approach provides a useful alternative to avoid overlooking relevant stationary points of the PES. ${ }^{1-4}$

The second possibility to examine unimolecular reactivity in mass spectrometry is to use chemical dynamics simulations by modeling the collisional system composed by the precursor ion and an inert gas atom $(\mathrm{Ar}, \mathrm{He}, \mathrm{Xe})$ or molecule $\left(\mathrm{N}_{2}\right.$ generally). ${ }^{5}$ After collision, the ion is internally activated, which eventually leads to fragmentation. Simulations provide reactivity in the short-time scale limit, since generally they run for some pico-seconds. This means that only fast processes, often non-statistical, are observed. This approach was pioneered by Hase and co-workers ${ }^{6,7}$ and it was successfully applied to a number of systems, from organic molecules ${ }^{8-12}$ to peptides ${ }^{13-15}$ and carbohydrates. ${ }^{16}$ Such an approach furnishes detailed information of the internal energy content of the ion after collisional activation; simulations also provide the partitioning between vibrational and rotational energy. While often only vibrational energy is considered in CID modeling, it has been shown that, in some cases, rotational activation is predominant. ${ }^{17,18}$

The simulations thus afford direct access to reaction products that occur in a very short time scale, and that are often produced by non- statistical mechanisms, i.e. before intramolecular vibrational relaxation (IVR) takes place. On the other hand, many of such trajectories do not react in the (short) simulation time but they have enough internal energy to react on longer time-scales. Assuming that these potentially reactive trajectories will react through a statistical mechanism, one can simulate their long time-scale behavior using RRKM theory. ${ }^{12,19,20}$

Recently, some of us have studied protonated uracil, [uracil] $\mathrm{H}^{+}, \mathrm{CID}$ by combining MS/MS experiments with chemical dynamics simulations of collisions. ${ }^{21}$ In this way, we have obtained detailed information on mechanisms occurring on fast time scales and explained the appearance of the different peaks observed experimentally. [Uracil] $\mathrm{H}^{+}$can have different tautomers, but the most stable one is the predominant in the gas phase, with a small abundance of the second most stable. ${ }^{22}$ Isomerization between them occurs on a time scale longer than that accessible by chemical dynamics simulations. Consequently, in our previous simulations we have used different tautomers as starting structures to investigate the correspondence between initial position of the proton and the products ions. $^{21}$

In the present study, we investigate the other limit of [uracil] $\mathrm{H}^{+}$ reactivity, i.e. the statistical reactivity. In particular, we employ an automated TS search protocol developed by one of us ${ }^{1,2}$ to find all possible fragmentation pathways and identify the corresponding TSs and minima. The reaction network thus created will be employed in subsequent RRKM and Kinetic Monte Carlo simulations that provide the statistical reactivity of the system.

Additionally, novel MS experiments are carried out here to study Ar + [uracil $] \mathrm{H}^{+}$fragmentation in the collision energy (in the center-ofmass reference frame) range 1-6 eV. The present statistical and 
experimental results are compared with previous chemical dynamics simulations. ${ }^{21}$ The comparison between theory and experiment is assisted by further $\mathrm{Ar}+$ [uracil] $\mathrm{H}^{+}$collisional dynamics simulations carried out here to investigate the efficiency of collisional energy transfer. In other words, the energy transfer results of the dynamics simulations provide a tool to turn $\mathrm{Ar}+[$ uracil $] \mathrm{H}^{+}$collisional energies into [uracil] $\mathrm{H}^{+}$internal energies. This way, the experimental product abundances can be compared with the statistical results.

\section{Computational details}

Automated transition state search method. An automated algorithm recently proposed by one of us ${ }^{1,2}$ is employed here to search for the stationary points of the $\mathrm{C}_{4} \mathrm{H}_{5} \mathrm{~N}_{2} \mathrm{O}_{2}{ }^{+}$system. The method is called TSSCDS and combines accelerated chemical dynamics simulations with the efficient BBFS (Bond Breaking/Formation Search) algorithm that searches for good TS candidates. These guess structures are then subjected to TS optimization employing the Eigenvector Following (EF) algorithm. Finally, Intrinsic Reaction Coordinate (IRC) calculations allow us connecting the obtained TSs with the different minima. The reader is referred to the Supplementary Information (SI) and to the original references for a detailed description of the method. ${ }^{1,2}$

TSSCDS involves several levels of electronic structure calculations. In the present work, the PM7 semiempirical method ${ }^{23}$ is employed for the direct dynamics simulations and to obtain initial TS structures, which are then re-optimized at the B3LYP/6-31+G(d,p) level of theory. The same DFT level is employed to run intrinsic reaction coordinate calculations (IRC), ${ }^{24}$ and to optimize the minima. The energies of all structures are corrected with B3LYP/6-311+G(3df,2p) single point calculations.

The PM7 calculations are carried out with MOPAC2012 25 and the DFT calculations are performed with GAUSSIAN0 $9^{26}$, both of which are interfaced with the TSSCDS program.

Finally, the structures of the stationary points are screened to remove duplicates and to categorize the minima as conformational isomers using topological coordinates, ${ }^{27}$ and properties of the adjacency and Laplacian matrices from spectral graph theory, ${ }^{28}$ as described in more detail in a previous paper. ${ }^{1}$

Kinetic calculations. The branching ratios of the different fragmentation pathways of protonated uracil are computed using a combination of both Rice-Ramsperger-Kassel-Marcus (RRKM) theory ${ }^{29}$ and Kinetic Monte Carlo (KMC) ${ }^{30,31}$ calculations. According to RRKM theory, the microcanonical rate coefficients $k_{i}(E)$ for an elementary step $i$ can be calculated as:

$k_{i}(E)=\sigma_{i} \frac{W_{i}^{T S}(E)}{h \rho_{i}(E)}$

where $\sigma_{i}$ is the reaction path degeneracy, $W_{i}^{T S}(E)$ is the sum of states at the TS, $\rho_{i}(E)$ is the density of states at the reactant, and $E$ is the excitation energy. The degeneracy of the reaction path is automatically generated thanks to an algorithm that detects symmetry elements from the molecular geometry. ${ }^{32}$ The conformational isomers are assumed to form a microcanonical ensemble, i.e., they are in equilibrium (see the ESI for further details). ${ }^{33}$ We have checked in previous work that this is a reasonable approach, provided the barriers between conformational isomers are relatively low. ${ }^{1,3}$ And this is indeed the case here where, e.g., the barrier for rotation about the $\mathrm{C} 4-\mathrm{O} 8 \mathrm{H}$ bond in MIN1 (see Figure 1) is only $0.4 \mathrm{eV}$.
Sums and densities of states are evaluated by direct count of the harmonic vibrational states using the Beyer-Swinehart algorithm. ${ }^{29}$ Since in this study reactivity was only observed for excitation energies greater than $\sim 3 \mathrm{eV}$, the energy range $3-30 \mathrm{eV}$ is employed for the kinetic calculations.

The RRKM rate coefficients computed as detailed above were subsequently employed in a KMC simulation ${ }^{30,31}$ to follow the transient behavior of the various molecular species that participate in the fragmentation of protonated uracil. For the RRKM and KMC calculations we employed programs from our research group.

Chemical Dynamics Simulations. Additional chemical dynamics simulations were performed to obtain the energy transfer due to collisions. To this end, we have considered a system composed by protonated uracil (in its most stable isomer) and a rare gas atom, Ar. The total potential energy of the collision system composed by protonated uracil and $\mathrm{Ar}$ is

$V=V_{[\text {uracil }] \mathrm{H}^{+}}+V_{\mathrm{Ar}}-[$ uracil $] \mathrm{H}^{+}$

where $V_{[\text {uracil] }} \mathrm{H}^{+}$is the intramolecular energy function of protonated uracil. In previous work we have shown that the reactivity is not significantly affected by the use of semiempirical or DFT direct dynamics to represent the intramolecular potential. ${ }^{21}$ On the other hand, the focus of the present dynamics simulations is on energy transfer, which depends more strongly on the interaction potential. For these reasons, we opted here to employ the PM3 semi-empirical Hamiltonian $^{34}$ to calculate the intramolecular potential $V_{[\text {uracil }] \mathrm{H}^{+}}$.

The second term of eq 2, $V_{\mathrm{Ar}-[\mathrm{uracil}] \mathrm{H}^{+}}$, is the interaction potential between the gas and the ion, which is obtained as a sum of two-body potentials:

$V_{\mathrm{Ar}-[\text { uracil }] \mathrm{H}^{+}}=\sum_{i} A_{A r-i} e^{-B_{A r-i} r_{A r-i}}+\frac{C_{A r-i}}{r_{A r-i}^{9}}$

where $i$ runs over all the [uracil] $\mathrm{H}^{+}$atoms. The parameters were obtained by fits to accurate quantum chemistry calculations as reported in the original works. ${ }^{7,21}$

The trajectories are initialized at the global minimum of protonated uracil using a quasi-classical $300 \mathrm{~K}$ Boltzmann sampling of rovibrational states. ${ }^{35,36}$ The ion is then randomly rotated about its Euler angles to take into account the random directions of the Ar+ [uracil] $\mathrm{H}^{+}$collisional system. The impact parameter is randomly sampled between 0 and $4.5 \AA$. This range is chosen to have a minimum energy transfer between $\mathrm{Ar}$ and [uracil] $\mathrm{H}^{+}$of at least $5 \%$ of the collision energy. Ar is collided against [uracil] $\mathrm{H}^{+}$at 13 different energies in the center-of-mass framework ranging from 1.3 to 11.3 $\mathrm{eV}$. For each collision energy ensembles of 10000 trajectories are employed to have reasonable statistics. The computer program VENUS96, ${ }^{37}$ coupled to MOPAC, ${ }^{38}$ is utilized to integrate the equations of motion. In particular, the standard velocity Verlet algorithm with a time step of $0.2 \mathrm{fs}$ is employed, which gives good energy conservation. The trajectories are initiated at an ion-projectile distance of $15 \AA$, for which the interaction between $\mathrm{Ar}$ and [uracil] $\mathrm{H}^{+}$ is negligible, and they are halted when the ion-projectile distance reaches $200 \AA$.

Mass Spectrometry Experiments. Mass spectrometry experiments were performed using an Applied Biosystems/MDS Sciex API2000 triple-quadrupole instrument fitted with a turboionspray source. 
Aqueous uracil solutions $\left(10^{-4} \mathrm{M}\right)$ were prepared with Milli-Q water and were introduced in the source using direct infusion with a syringe pump at a flow rate of $5 \mu 1 / \mathrm{min}$. The sample was ionized by applying a voltage of $5.5 \mathrm{kV}$ on the sprayer probe and by the use of a nebulizing gas (GAS1, air) surrounding the sprayer probe, perpendicularly intersected by a heated gas (GAS2, air). Pressure sensors were used to adjust the operating pressure of GAS1 and GAS2 to 2.1 bars, as a fraction of the air inlet pressure. To prevent the access of air or solvent into the analyzer region, $\mathrm{N}_{2}$ (curtain gas) was used at a pressure of 1.4 bar. The temperature of GAS2 was set to $100^{\circ} \mathrm{C}$. CID spectra were recorded by introducing nitrogen as collision gas in the second quadrupole. Low gas pressures were used to limit multiple ionmolecules collisions and the declustering potential was set to $40 \mathrm{~V}$. CID spectra were recorded in the collisional energy (in the laboratory frame) range 5-30 eV with a $5 \mathrm{eV}$ step, which corresponds to collision energies ranging from 1 to $6 \mathrm{eV}$ in the center-of-mass (com) frame. In the following, collision energies will always refer to the com frame. We should also note that the different collision partners employed in the experiments (nitrogen) and in the simulations (argon) might give rise to different energy transfer efficiencies (greater for $\mathrm{Xe} / \mathrm{Kr}$ than for Ar than for $\left.\mathrm{N}_{2}\right) .{ }^{17,39}$

Since MS/MS spectra are very likely obtained under a multiplecollision regime, the internal energy content of the precursor ion is increased. Setting the CAD parameter (which controls the amount of nitrogen introduced into Q2) to its minimum value, the pressure value measured by the ion gauge, located at the vicinity of Q2, is about $3 \times 10^{-5}$ Torr, but the actual pressure inside Q2 cannot be determined accurately. However, it has been reported that the pressure inside the collision cell is in fact in the order of $10 \mathrm{mTorr}^{40}$ At this pressure value and taking into account the dimensions of Q2, gas kinetic theory estimates a mean free path of several $\mathrm{mm}$ for a moving $\mathrm{N}_{2}$ molecule. This means that a $\mathrm{N}_{2}$ molecule may undergo tens of collision in its way through Q2. This is considered as a lower limit for uracil, which has a larger collision cross section for being bigger than $\mathrm{N}_{2}$. Uracil was purchased from Aldrich (Saint-Quentin Fallavier, France) and used without further purification.

\section{Results and discussion}

Stationary points. A total of 1398 stationary points (647 minima and $751 \mathrm{TSs}$ ) have been found in this study in the ground electronic state of the $\mathrm{C}_{4} \mathrm{H}_{5} \mathrm{~N}_{2} \mathrm{O}_{2}{ }^{+}$system in the DFT potential energy surface. The structures are tagged (with MIN for minimum and TS for transition state) and sorted according to their relative energies (including the ZPE). The 647 minima include protonated uracils (see Figure 1), as well as five member rings and open-extended structures.

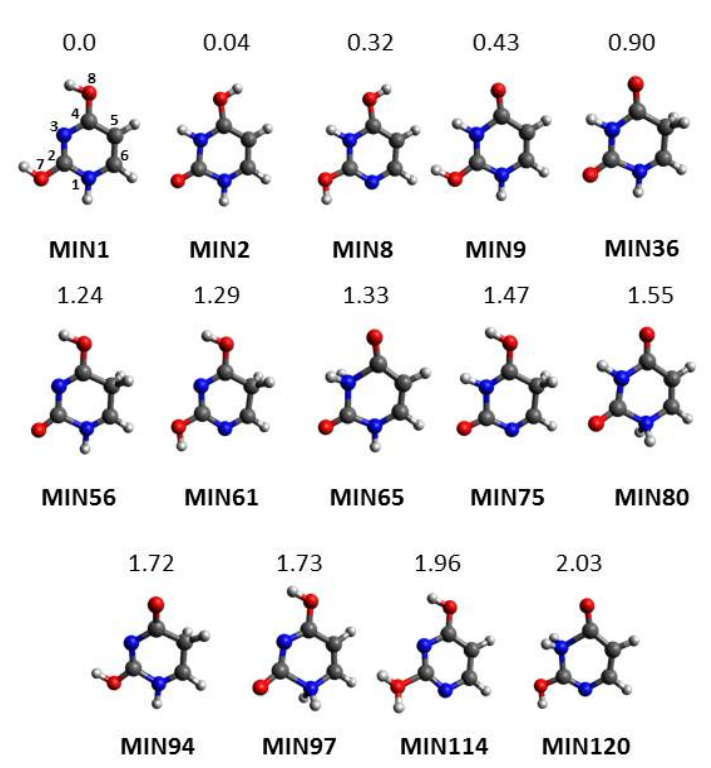

Figure 1. Lowest-energy protonated forms of uracil found in this study. The structures are optimized at the B3LYP/6-31+G(d,p) level of theory. The numbers are relative energies in $\mathrm{eV}$ calculated at the B3LYP/6-311+G(3df,2p)//B3LYP/6$31+\mathrm{G}(\mathrm{d}, \mathrm{p})$ level of theory with the zero-point energy correction obtained by B3LYP/6-31+G(d,p) frequency calculations. The classical numbering used for uracil is shown for the lowest energy structure MIN1.

In previous work, ${ }^{21}$ a total of six protonated forms of uracil were considered. Figure 1 shows the 14 lowest-energy structures of protonated uracil obtained in this work; only the lowest-energy conformational isomer of each structure is depicted. The structures of the previous study correspond to minima $1,2,9,65$, 80 and 120 of Figure 1.

The transition states either connect different isomers of the system, correspond to bimolecular processes, or lead to the following fragments: molecular hydrogen $\left(\mathrm{H}_{2}\right)$, carbon monoxide $(\mathrm{CO})$, water $\left(\mathrm{H}_{2} \mathrm{O}\right)$, hydronium $\left(\mathrm{H}_{3} \mathrm{O}^{+}\right)$, carbon dioxide $\left(\mathrm{CO}_{2}\right)$, protonated carbon dioxide $\left(\mathrm{CO}_{2} \mathrm{H}^{+}\right)$, hydrogen cyanide $(\mathrm{HCN})$, protonated hydrogen cyanide $\left(\mathrm{HCNH}^{+}\right)$, hydrogen isocyanide (HNC), nitroxyl (HNO), formyl radical $(\mathrm{HCO})$, cyanic acid $(\mathrm{NCOH})$, protonated cyanic acid $\left(\mathrm{H}^{+} \mathrm{NCOH}\right)$, isocyanic acid $(\mathrm{HNCO})$, protonated isocyanic acid $\left(\mathrm{HNCOH}^{+}\right)$, ethylene $(\mathrm{HCCH})$, formaldehyde $\left(\mathrm{CH}_{2} \mathrm{O}\right)$, ammonia $\left(\mathrm{NH}_{3}\right)$, formic acid $(\mathrm{HCOOH})$, protonated formic acid $\left([\mathrm{HCOOH}] \mathrm{H}^{+}\right)$, ketene $\left(\mathrm{H}_{2} \mathrm{CCO}\right)$, ethynol, $(\mathrm{HCCOH})$, cyanomethyl radical $\left(\mathrm{CH}_{2} \mathrm{CN}\right)$, methaniminium cation $\left(\mathrm{CH}_{2} \mathrm{NH}_{2}^{+}\right)$, and bigger fragments.

Some TSs have also been found to lead to triple dissociations (3 different products), where more than one of the above fragments are formed in the same channel. A complete description of the dissociation channels is given in the SI, as well as a detailed comparison between the PM7 and DFT geometries and energies for the $751 \mathrm{TSs}$. 

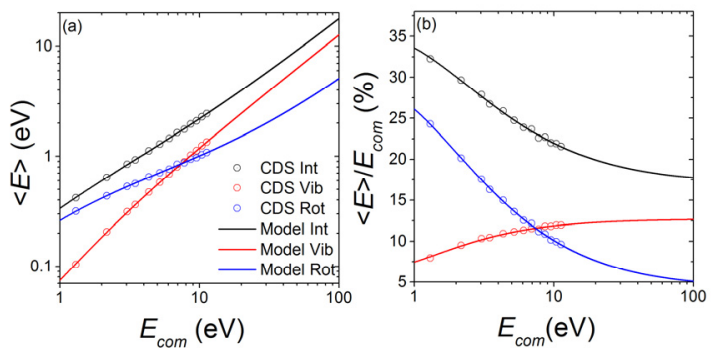

Figure 2. (a) Average (internal, vibrational and rotational) energies $\langle E\rangle$ of [uracil] $\mathrm{H}^{+}$as a function of the collision energy $E_{\text {com }}$ (in the center-of-mass framework) after colliding with Ar. (b) Percent energy transfer values to [uracil $] \mathrm{H}^{+}$internal degrees of freedom. Symbols and solid lines correspond to the chemical dynamics simulation (CDS), and energy transfer model results, respectively.

Energy Transfer. In a non-elastic scattering event, as occurring in CID experiments, the amount of energy transferred to the ion's internal degrees of freedom, and the ratio between vibrational and rotational activation of the ion after collision, are a priori not known. Chemical dynamics simulations can provide such information.

Note that in the present simulations the maximum impact parameter is $4.5 \AA$, a value much larger than that used in the previous simulations $(0.5 \AA),{ }^{21}$ resulting in a dramatic decrease of reactivity. In fact, no reactive events were found in the present dynamics simulations, which are only meant to provide detailed energy transfer results. By contrast, our previous simulations were designed to study reactivity, which anyway turned out to be modest: less than $1 \%$ of the trajectories were found to be reactive when using MIN1 as initial structure. ${ }^{21}$

Figure $2 \mathrm{a}$ shows the average (internal, vibrational and rotational) energies of [uracil] $\mathrm{H}^{+}$after colliding with Ar. The final internal energies are a relatively small fraction of the collision energy as shown in Figure $2 \mathrm{~b}$ where, for instance, at the highest collision energy of $11.3 \mathrm{eV}$ the internal energy of protonated uracil is slightly below $2.5 \mathrm{eV}$ (Figure 2a), which corresponds to a percentage of 22\% (Figure $2 b)$.

The chemical dynamics results obtained in the $1.3-11.3 \mathrm{eV}$ collision energy range can be extrapolated to higher collision energies using a phenomenological energy transfer model recently proposed by one of us. ${ }^{41,42}$ The model is an adaptation of two limiting models of energy transfer to atom-diatom collisions, and it was successfully applied to a number of systems. ${ }^{12,41,42}$ In the model, the average energy $\langle\Delta E\rangle$ transferred to rotation or vibration of the ion is given by:

$\langle\Delta E\rangle=a_{1} \exp \left(\frac{-b_{1}}{\sqrt{E_{c o m}}}\right)+a_{2} \operatorname{cosech}^{2}\left(\frac{b_{2}}{\sqrt{E_{c o m}}}\right)$

with $a_{1}, a_{2}, b_{1}$, and $b_{2}$ being adjustable parameters. The above equation predicts a limiting high collision-energy value of $a_{2} / b_{2}{ }^{2}$ for the fraction $\langle\Delta E\rangle / E_{\text {com }}$. The fits of eq (4) to the chemical dynamics energy transfer results provide the following values for the parameters: $a_{1}=0.05 \mathrm{eV}, a_{2}=0.42 \mathrm{eV}, b_{1}=0.62 \mathrm{eV}^{1 / 2}$ and $b_{2}=$ $1.81 \mathrm{eV}^{1 / 2}$ for vibrational energy transfer, and $a_{1}=0.97 \mathrm{eV}, a_{2}=$ $0.16 \mathrm{eV}, b_{1}=1.37 \mathrm{eV}^{1 / 2}$ and $b_{2}=1.95 \mathrm{eV}^{1 / 2}$ for rotational energy transfer.
Interestingly, for collision energies lower than $7.5 \mathrm{eV}$ rotational activation dominates, but for higher collisional energies, transfer to vibration becomes increasingly more important. This is clearly seen in Table 1, where the percent energy transfer to rotation is $24 \%$ for $E_{\text {com }}=1.3 \mathrm{eV}$ compared to a modest $8 \%$ transfer to vibration. However, in the high collision energy limit, the percent transfer to vibration is nearly three-fold that to rotation. Previous CID studies have also shown a predominance of $T \rightarrow R$ energy transfer, ${ }^{43}$ a result that can be explained by the planar shape of the ion. A previous CID study on the $\mathrm{Xe}+[$ uracil $] \mathrm{Li}^{+}$system shows energy transfer efficiencies in line with those obtained here. ${ }^{12}$

The substantial amount of rotational excitation at low collision energies is a relevant result because RRKM rate constants depend upon the amount of rotational activation of the system. ${ }^{44}$ However, for the range of energies where rotational activation is predominant, we have not observed reactivity in the present study (vide infra). The fragmentation mechanisms found here are only accessible at relatively high internal energies of the ion (greater than $\sim 3 \mathrm{eV}$ ), where the system is primarily vibrationally activated (see Figure $2 \mathrm{a}$ ). For that reason, only vibrational densities and sums of states are considered in the RRKM calculations of the present study.

Table 1: Percent energy transfer to the internal degrees of freedom (dof) of [uracil] $\mathrm{H}^{+}$after collision with Ar.

\begin{tabular}{|c|c|c|c|c|c|}
\hline \multirow{2}{*}{ dof } & \multicolumn{5}{|c|}{$E_{\text {com }}(\mathrm{eV})$} \\
\cline { 2 - 6 } & $1.3^{\mathrm{a}}$ & $10^{\mathrm{a}}$ & $20^{\mathrm{b}}$ & $30^{\mathrm{b}}$ & $\infty^{\mathrm{b}}$ \\
\hline Vibration & 8.0 & 11.9 & 12.3 & 12.4 & 12.7 \\
\hline Rotation & 24.0 & 10.0 & 7.6 & 6.6 & 4.3 \\
\hline
\end{tabular}

${ }^{\text {a}}$ From chemical dynamics simulations. ${ }^{b}$ Extrapolated values using the energy transfer model.

It is also important to note that, even though the average internal energies of protonated uracil are modest as seen above, the energy distributions present tails that extend up to much larger energies. For instance, at $E_{\text {com }}=11.3 \mathrm{eV}$ up to $35 \%$ of the internal energy distribution corresponds to energies greater than $3 \mathrm{eV}$ (see Figure $2 \mathrm{~S}$ of the SI), which is the threshold for statistical reactivity (vide infra). In other words, when the collision energy of the $\mathrm{Ar}+[$ uracil $] \mathrm{H}^{+}$ system is $11.3 \mathrm{eV}$, according to the present results, $65 \%$ of the trajectories would be unreactive, and the remaining $35 \%$ would decompose according to the fragmentation channels described below.

The percentage of trajectories with internal energies greater than $3 \mathrm{eV}$ diminishes to $15 \%$ and $2 \%$ for $E_{\text {com }}=6.06$ and $4.34 \mathrm{eV}$, respectively. For lower collision energies the final internal energies of all trajectories are below $3 \mathrm{eV}$. These energy distributions, as well as the KMC product abundances discussed in the next section, are employed to calculate the weighted statistical results that will be discussed later.

Fragmentation channels. The kinetic simulations carried out in this study in the energy range $3-30 \mathrm{eV}$ lead to the product abundances displayed in Figure 3. For the sake of completeness, we provide here kinetic results for uracil's excitation energies up to $30 \mathrm{eV}$, even though the highest collision energy (in the centerof-mass frame) of our MS experiments is only $6 \mathrm{eV}$. 
The KMC simulations show that fragmentation of protonated uracil primarily leads to the following dissociation fragments (only the channels with yields greater than $0.2 \%$ are listed): isocyanic acid $(\mathrm{HNCO})$, ammonia $\left(\mathrm{NH}_{3}\right)$, water $\left(\mathrm{H}_{2} \mathrm{O}\right)$, protonated isocyanic acid $\left(\mathrm{HNCOH}^{+}\right)$, carbon monoxide $(\mathrm{CO})$, acetylene $(\mathrm{HCCH})$, molecular hydrogen $\left(\mathrm{H}_{2}\right)$, and hydrogen isocyanide (HNC).

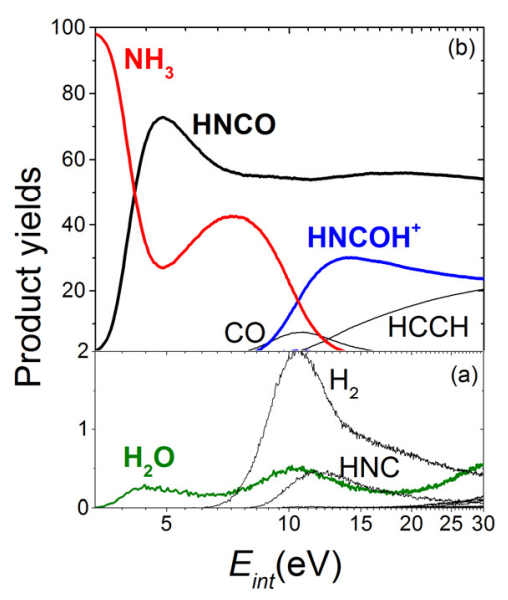

Figure 3. Product yields of the different fragmentation channels of protonated uracil obtained in the KMC simulation as a function of internal energy content $E_{\text {int }}$ in the molecule. Plots (a) and (b) show product abundances in the ranges $0-2$ and $2-100 \%$, respectively. The simulation results extend up to $30 \mathrm{eV}$ even though the present MS experiment only access protonated uracil's internal energies of $\sim 7.6 \mathrm{eV}$.

Overall, the major channels obtained in the present study coincide with those found previously. ${ }^{21}$ Figure 4 shows the major fragmentation channels obtained in the present MS/MS experiments in the 1-6 eV collision energy range. They will be discussed in detail and compared with our statistical results in a separate section below.

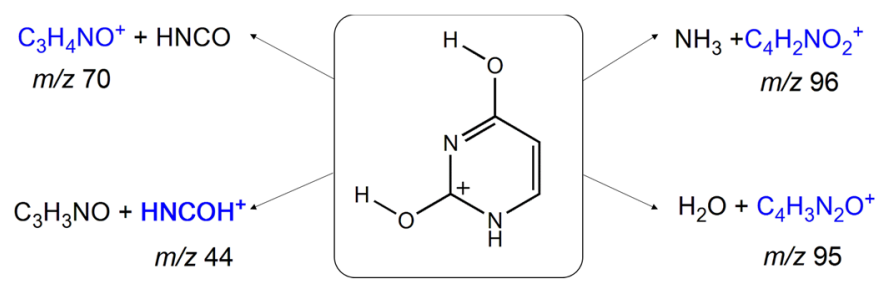

Figure 4. Major fragmentation channels obtained in our MS/MS experiments.

The HNCO fragment corresponds to the $m / z 70$ peak in the CID mass spectrum (see Figure 4), and it was observed in all the MS/MS experiments of [uracil] $\mathrm{H}^{+}$reported so far. Additionally, the $\mathrm{NH}_{3}, \mathrm{H}_{2} \mathrm{O}, \mathrm{HNCOH}^{+}, \mathrm{HCCH}, \mathrm{CO}, \mathrm{H}_{2}$ and $\mathrm{HNC}$ channels would appear in the mass spectrum at the following $\mathrm{m} / \mathrm{z}$ values: 96, 95, 44, 87, 85, 111 and 86, respectively. The $\mathrm{m} / \mathrm{z} 96,95$ and 44 ions were clearly observed in experiments (see Figure 4). ${ }^{21}$ By contrast, $m / z$ 85, 86, 87 and 111 ions were not detected in experiments done by Nelson and McCloskey, ${ }^{45}$ Beach and Gabryelski ${ }^{46}$ and our group, ${ }^{21}$ while a $\mathrm{m} / \mathrm{z} 85$ ion (corresponding to CO loss) was reported by Sadr-Arani et al. ${ }^{47}$ Note that we have also obtained the $\mathrm{m} / \mathrm{z} 85$ ion in some of the direct dynamics of fragmentation. ${ }^{21}$ The simulations, actually, show that this product is unstable and can easily dissociate further, which might explain the absence of any peak in that region of the MS spectra.

The lack of any signal in the MS spectra at $m / z 86,87$ and 111 could also be explained by further fragmentation of these ions. Additionally, the absence of the $\mathrm{m} / \mathrm{z} 87$ peak (loss of acetylene) in the experiments can have another explanation: our results show that acetylene is only formed at internal energies greater than 10-12 eV (see Figure 3), which corresponds to collision energies $(\sim 80-100 \mathrm{eV}$ as seen in Figure $2 \mathrm{a})$ greater than those employed in our MS experiments. Finally, the onset of $\mathrm{H}_{2}$ and HNC products formation ( $\mathrm{m} / \mathrm{z} 86$ and 111 peaks, respectively) also appears at relatively high internal energies (see Figure 3 ) and their yields are very modest, which makes it difficult to detect experimentally.

In the following we discuss the fragmentation pathways corresponding to the most important reaction channels.

HNCO channel. This fragmentation channel leads to the formation of the neutral species $\mathrm{HNCO}$ and the $m / z 70$ ion, which is one of the most intense peaks of the reported [uracil] $\mathrm{H}^{+} \mathrm{MS} / \mathrm{MS}$ spectra. $^{21,45}$ The most relevant mechanisms leading to this product are shown in Figure 5, in terms of minima and transition states connecting different isomers or an isomer with the products.

The lowest-energy pathway (purple; Figure 5a) is initiated by a proton transfer (PT) from the O7-H group to N3 leading to MIN2. Then, from MIN2 another PT leads to MIN65. This process weakens the N3-C4 bond, which ultimately breaks, forming the open-extended structure MIN29. Finally, the $\mathrm{NH}_{2}{ }^{+}$ group transfers a proton, forming the neutral species $\mathrm{HNCO}$ and the ion at $m / z 70$.

Alternatively, a PT from the O8-H group to N3 forms MIN9 (red; Figure 5b), which can undergo cleavage of the N3-C4 bond leading to MIN 74. Finally, another PT from the O7-H group with a concomitant $\mathrm{C} 2-\mathrm{N} 1$ bond breakage leads to $\mathrm{HNCO}$ and the $m / z 70$ ion.

The shortest pathway (blue; Figure 5c) involves only two steps: (a) formation of the open-extended structure MIN15, after PT from the $\mathrm{O} 7-\mathrm{H}$ group to $\mathrm{N} 1$, and (b) concerted $\mathrm{N} 3-\mathrm{C} 4$ bond breakage and $\mathrm{PT}$ from the $\mathrm{O} 8 \mathrm{-H}$ group to $\mathrm{N} 3$ giving rise to the final products.

Finally, another route (green; Figure 5d) involving several PT processes, and cleavage of the $\mathrm{C} 4-\mathrm{C} 5$ and N3-C2 bonds leads to a higher energy isomer of the $\mathrm{m} / \mathrm{z} 70$ ion and $\mathrm{HNCO}$.

In all the above HNCO loss pathways, the rate limiting step is a PT process. We should also note that these minimum energy pathways do not involve any concerted bond breaking process, as in the Retro Diels-Alder (RDA) mechanism proposed by Nelson and McCloskey ${ }^{45}$ and observed in our previous direct dynamics simulations. ${ }^{21}$ Rather, these stepwise pathways are more similar to the mechanisms proposed by Beach and Gabryelski. ${ }^{46}$ 
There are (at least) three possible reasons that might explain the lack of TSs for the RDA mechanism:

(1) The RDA mechanism might proceed via a "loose" TS. Variational RRKM theory would give us in this case the exact location of the TS via minimization of the sum of states along the reaction coordinate.

(2) The RDA mechanism might be a consequence of non-IRC dynamics.
(3) Our TS search algorithm might fail to find the RDA TS. In our opinion this is not very likely, as multiple manual searches for the RDA TS, carried out here and in our previous work, ${ }^{21}$ failed.

Inspection of our previously reported direct dynamics simulations shows that the RDA mechanism is a nonsynchronous concerted mechanism.
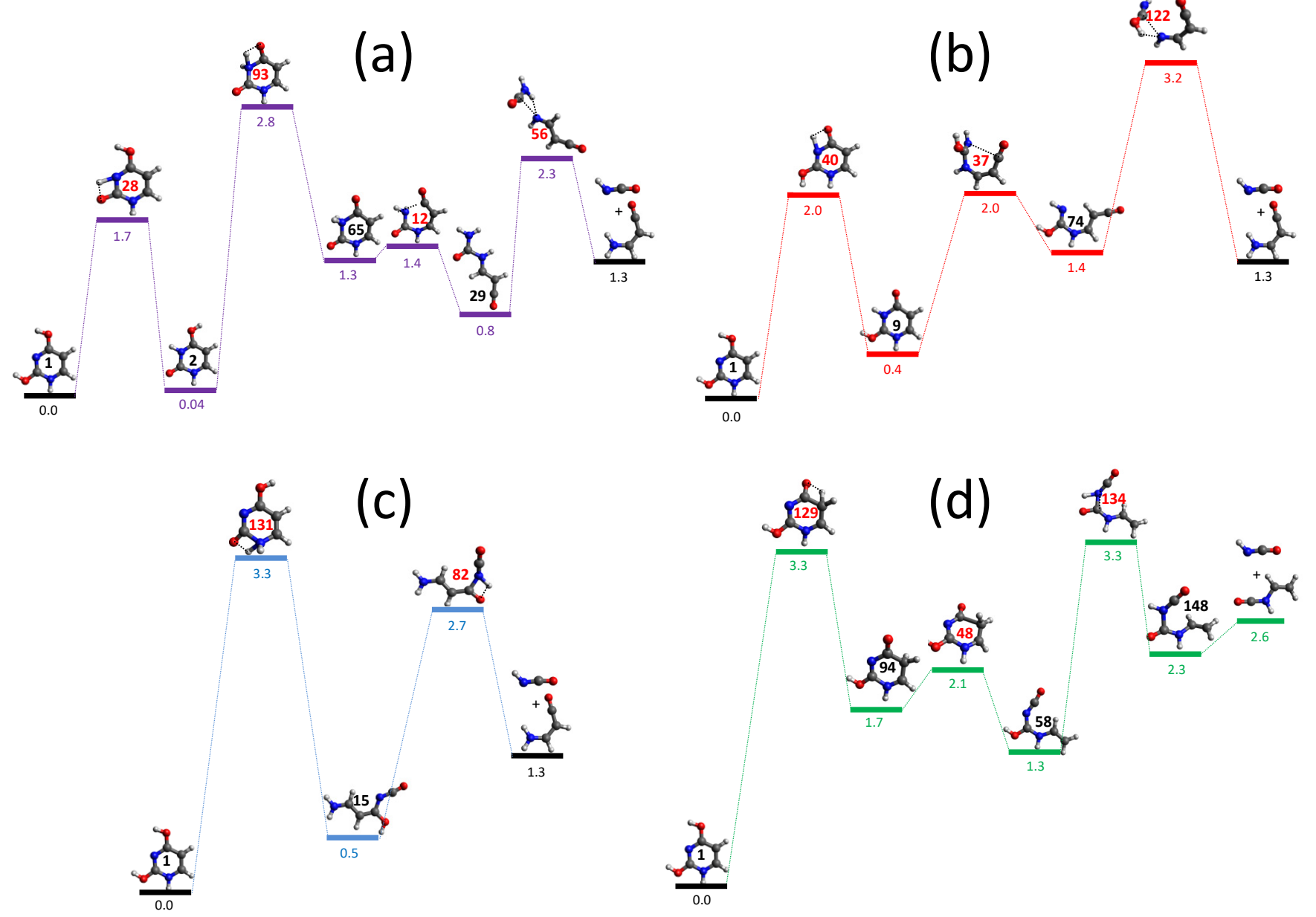

Figure 5. Most relevant (in terms of kinetics) fragmentation pathways leading to HNCO. A complete description of all channels is collected in the ESI. Relative energies expressed in eV. The labels of the minima are in black and those of the TSs in red. Colors are also employed to differentiate the various fragmentation channels. Note that barriers between conformational isomers are very low and, consequently, they are not depicted in the figures for clarity, and also because they are not relevant in the kinetics study (see the text and ESI for further details). Also, the different mechanisms can be connected by different TSs (not shown for clarity), like TS 114 connecting minima 2 and 15 (involved in the purple and blue mechanisms, respectively, TS 105 connecting minima 65 and 9 (involved in the purple and red mechanisms, respectively), or TS 143 connecting minima 29 and 74 (involved in the purple and red mechanisms, respectively). 


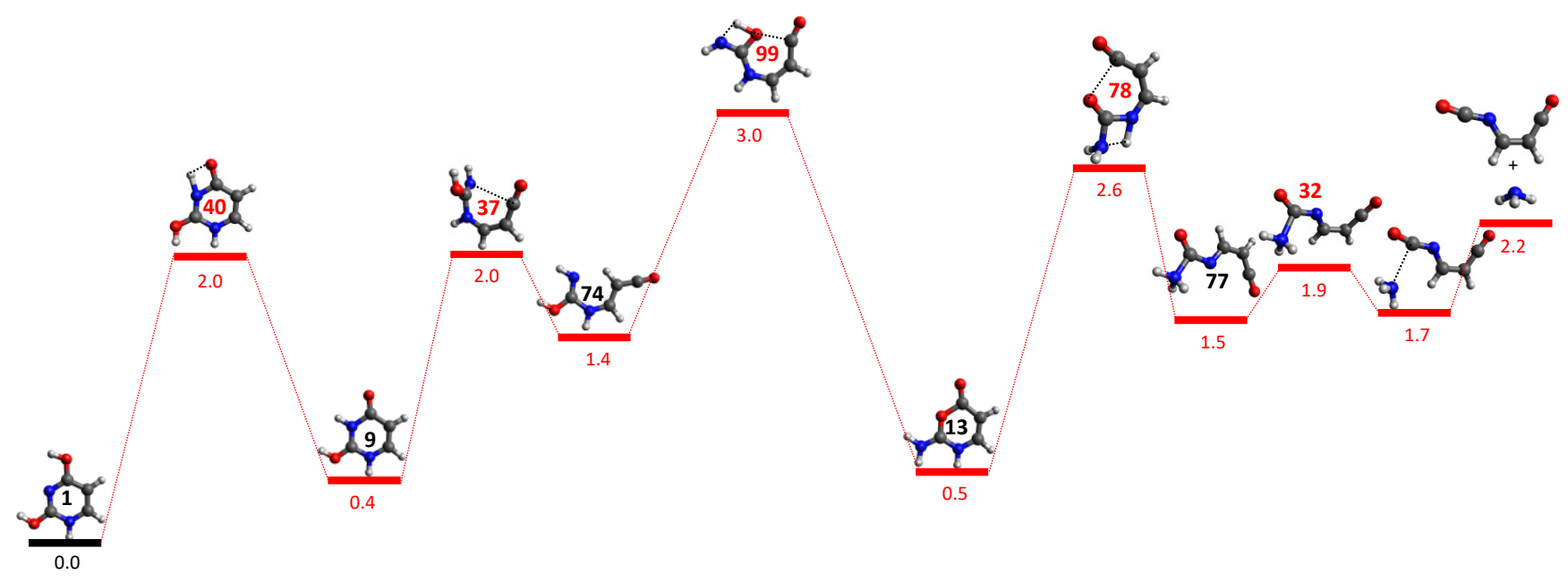

Figure 6. Diagram showing the fragmentation pathway leading to $\mathrm{NH}_{3}$. Relative energies expressed in eV. The labels of the minima are in black and those of the TSs in red. An alternative (and longer) route connecting MIN 1 and MIN 74 was found through minima 2, 65 and 29 (see the ESI).

NH$_{3}$ channel. The automated procedure employed in the present study only provided one mechanism for ammonia loss (see Figure 6). The unimolecular reactivity is, again, initiated by a PT from the O8H group to N3, which leads to the formation of MIN9. Then, the N3$\mathrm{C} 4$ bond is cleaved, leading to the formation of MIN74. A longer route to MIN74 can be traced via isomers MIN2, MIN65 and MIN29, but it was not shown in the figure for clarity.

From MIN74, a second proton is transferred from the O7-H group to N3 with a concomitant bond formation, leading to the cyclic structure MIN13. From this isomer, ring-opening and PT from the N1-H group to N3 occur concertedly, forming the extended-open structure MIN77, which finally leads to ammonia and the $m / z 96$ ion after surmounting a relatively small barrier.

The $m / z 96$ peak is also characteristic of the [uracil] $\mathrm{H}^{+} \mathrm{MS} / \mathrm{MS}$ spectrum. Moreover, isotopic labeling experiments ${ }^{21,45}$ have shown that the $\mathrm{N} 3$ atom is involved in the $\mathrm{NH}_{3}$ loss, in agreement with the present statistical calculations. We should also note that these mechanisms are similar to those observed in our previous direct dynamics simulations of fragmentation. ${ }^{21}$

$\mathrm{HNCOH}^{+}$channel. Another product observed in both experiments and previous simulations is $\mathrm{HNCOH}^{+}$, which corresponds to the $\mathrm{m} / \mathrm{z}$ 44 ion in the spectra. As seen in the SI, only two pathways were found leading to this product. The one corresponding to the lowest energy is shown in Figure 7.

In this case, the reactivity is not initiated by any proton transfer Rather, the cycle is opened and, in a subsequent step, a proton is taken by the leaving group forming the product $m / z 44$ ion. This mechanism is not exactly concerted but it is very close to a concerted reaction and thus to a Retro Diels Alder mechanism, which was observed for this channel in our previous dynamics simulations (even starting from different uracil isomers). ${ }^{21}$

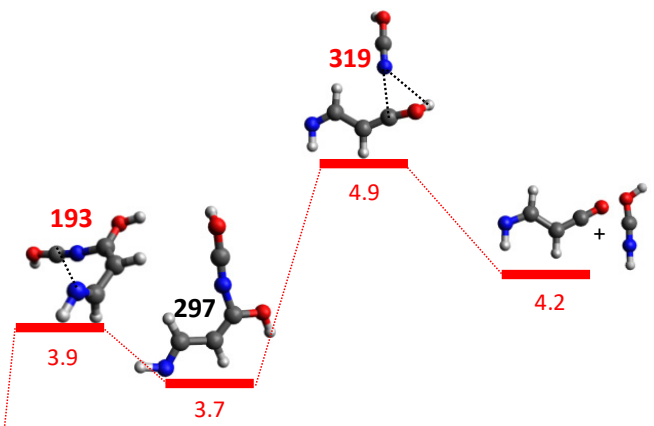

Figure 7. Diagram showing the lowest-energy fragmentation pathway leading to $\mathrm{HNCOH}^{+}$. A complete description of all channels is collected in the ESI. Relative energies expressed in $\mathrm{eV}$. The labels of the minima are in black and those of the TSs in red. 
$\mathrm{H}_{2} \mathrm{O}$ channel. The four most important mechanisms leading to $\mathrm{H}_{2} \mathrm{O}$ and the $m / z 95$ ion are shown in Figure 8. They are all initiated by the MIN1 $\rightarrow$ MIN9 isomerization process (via TS 40) that is common to the $\mathrm{HNCO}$ and $\mathrm{NH}_{3}$ channels and it is not shown again in Figure 8 for simplicity.

The shortest pathway (depicted in red in Figure 8a) involves only two steps. The first one is the above-mentioned MIN1 $\rightarrow$ MIN9 isomerization, while the second one occurs via a four-centered elimination TS (TS155), which leads to water and the $m / z$ 95 ion.

The lowest-energy pathway (purple in Figure 8b) occurs via MIN74, which was also involved in the HNCO and ammonia loss pathways. From that structure, the $\mathrm{O} 7-\mathrm{H}$ group is transferred from $\mathrm{C} 2$ to $\mathrm{C} 4$ leading to MIN123, which undergoes a concerted proton transfer and $\mathrm{C}-\mathrm{O}$ bond cleavage forming water and the $\mathrm{m} / \mathrm{z} 95$ ion.

The two pathways depicted in panels c and d of Figure 8 (blue and green, respectively) also involve MIN74. The water elimination pathway shown in blue (Figure 8c) occurs via the four-centered transition state TS185, whereas the green pathway (Figure 8d) involves two consecutive PTs, resulting in the formation of water and the $m / z 95$ ion.

It is important to remark that in all the mechanisms found in the present study, water is formed only from one of the oxygens $(\mathrm{O} 7)$, whereas in the experiments and also in our previous direct dynamics simulations, ${ }^{21,}{ }^{45}$ both oxygen atoms were found in the resulting $\mathrm{H}_{2} \mathrm{O}$ molecules.

Note that the shortest pathway obtained here for $\mathrm{H}_{2} \mathrm{O}$ loss (Figure 8a) is very similar (although not identical) to the mechanism proposed in our previous collision dynamics study. ${ }^{21}$ This suggests that some water loss occurs in a statistical way, while other processes follow non-statistical behavior. As we will discuss in the next section nonstatistical processes are probably very important, since the abundance of $\mathrm{H}_{2} \mathrm{O}$ loss obtained in the present $\mathrm{KMC} / \mathrm{RRKM}$ simulations is much lower than that observed experimentally.

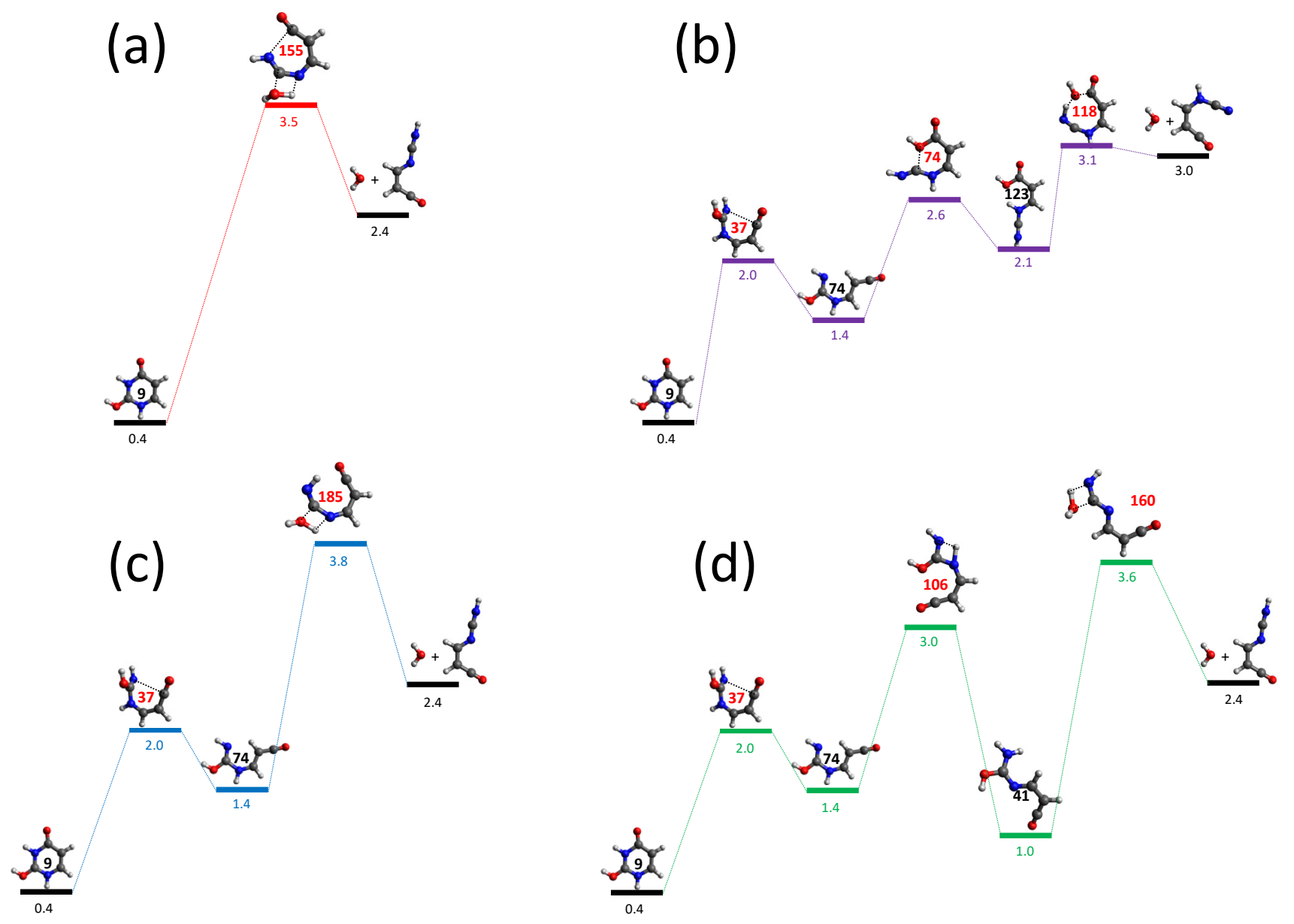

Figure 8. Most relevant (in terms of kinetics) fragmentation pathways leading to $\mathrm{H}_{2} \mathrm{O}$. A complete description of all channels is collected in the ESI. Relative energies expressed in eV. The labels of the minima are in black and those of the TSs in red. Colors are also employed to differentiate the various fragmentation channels. 
Comparison with experiments. Figure 9a shows the experimental abundance of ions after MS/MS of [uracil] $\mathrm{H}^{+}$("precursor ion" in the figure) as a function of the collision energy in the center-of-mass framework. The main peaks at all energies are $m / z 70$ (HNCO loss), $m / z 96\left(\mathrm{NH}_{3}\right.$ loss$)$ and $m / z 95\left(\mathrm{H}_{2} \mathrm{O}\right.$ loss $)$.

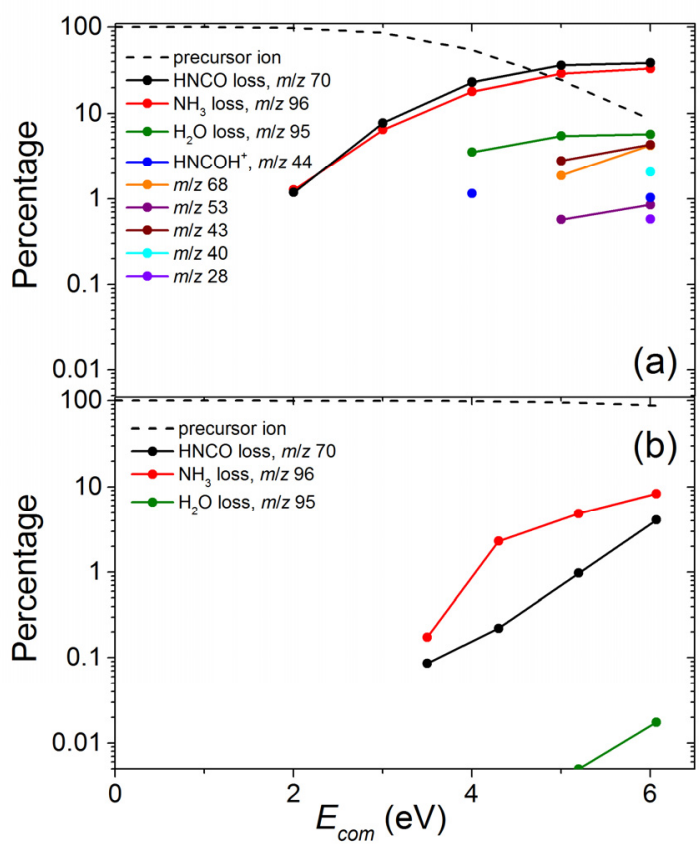

Figure 9. (a) Experimental intensities of precursor and fragment ions generated upon CID of protonated uracil as a function of the collision energy $E_{\text {com }}$ (in the center-of-mass framework). (b) Theoretical intensities of precursor and fragment ions obtained in this study as a function of the collision energy $E_{\text {com }}$.

Experimental and statistical intensities of the precursor and fragment ions can be compared using the energy transfer results from the dynamics simulations alongside with the theoretical product abundances of Figure 3. In particular, the ion abundances of Figure 3 have been weighted using the energy transfer probabilities obtained in this study at the different collision energies. The results are shown in Figure $9 b$.

For very low collision energies $(\sim 2 \mathrm{eV})$ the MS/MS experiments show how the $\mathrm{m} / \mathrm{z} 96$ ion is slightly more abundant than $\mathrm{m} / \mathrm{z} 70$, although, in general, the intensity of both peaks is pretty similar over the whole collisional energy range (Figure 9a). On the other hand, our theoretical results show that ammonia loss is predominant, particularly at low collision energies (Figure 9b), although the abundances of both ions reach a similar value (around 15\%) for the highest collision energy employed in the dynamics simulations (11.3 $\mathrm{eV})$.

Overall, the fragment ion abundances found in our weighted statistical calculations are lower than those found in the MS/MS experiments, particularly at very low collision energies. Reactivity at such low collision energies can have two origins: (i) fast "non-statistical" processes, which are not considered in the present statistical study, or (ii) multiple collisions in the MS/MS experiments. Since no reactivity was observed in the collisional simulations for $E_{\text {com }}=3 \mathrm{eV}$, the first possibility would result from a rare event. Multiple collisions, on the other hand, are possible, resulting in higher final internal activation energy.
In our experiments, $\mathrm{H}_{2} \mathrm{O}$ loss $(\mathrm{m} / \mathrm{z} 95)$ is the third most abundant product, which agrees with the statistical theoretical results, although the theoretical abundance for this ion is $\sim 10^{2}$-fold lower than the experimental value. Note that in our previous work where fragmentation occurred in the fast time scale limit (i.e. mainly nonstatistical conditions for unimolecular fragmentation), this product was obtained with higher abundance than in the present study. ${ }^{21}$ Furthermore, the present statistical calculations identify water loss pathways where only one of the oxygen atoms is involved (see Figure 8), while previous experiments and simulations have shown that both oxygens are found in the $\mathrm{H}_{2} \mathrm{O}$ product. This suggests that water loss, most likely, proceeds via a non-statistical process.

Finally, $\mathrm{HNCOH}^{+}(\mathrm{m} / \mathrm{z} 44)$ is a relatively important product in $\mathrm{KMC}$ simulations (see Figure 3), but it is only formed at internal energies greater than $8 \mathrm{eV}$, which explains why this product presents a very low abundance in the experiments (around 1\%) and also in the weighted statistical results (its abundance is $\sim 0.0001 \%$ and therefore it does not appear in Figure 9b). Our energy transfer results show that, even at the highest collision energy of $11.3 \mathrm{eV}$, only a negligible fraction of trajectories present internal energies around $8 \mathrm{eV}$.

\section{Conclusions}

1. Using an automated TS search method, up to 647 isomers of protonated uracil are found in the present work, including ring and open-extended structures. Besides, the method provides 751 TSs and their corresponding reaction pathways, including isomerizations between pairs of isomers, unimolecular fragmentations and bimolecular reactions.

2. The energy transfer analysis indicates that, at the collision energies employed in the MS experiments, only relatively low $(<5 \mathrm{eV})$ internal energies of protonated uracil are readily accessible. An energy transfer model is employed to extrapolate the energy transfer results to higher collision energies.

3. For the ion's internal energies reachable in the experiments, the KMC simulations show that the major fragmentation channels are isocyanic acid and ammonia losses, in good agreement with experiments. According to both KMC simulations and experiments, these two channels are followed by water loss, although its simulated yield is very low compared with the experiments, suggesting that non-statistical dynamics might play an important role for this channel.

4. Isocyanic acid $\left(\mathrm{HNCOH}^{+}\right)$is also an important product in the KMC simulations, although only at relatively high internal energies $(>8 \mathrm{eV})$. These energies cannot be reached using the range of collision energies of the experiments, which explains the low signal observed for this ion in the MS experiments and in the weighted statistical results.

5. Several channels leading to carbon monoxide and the $\mathrm{m} / \mathrm{z} 85$ ion have been obtained in the present theoretical study, and they compete with the other fragmentations for internal energies in the range $8-15 \mathrm{eV}$. The lack of any signal at $\mathrm{m} / \mathrm{z} 85$ in the current MS experiments can be explained by further fragmentations of the ion as it 
was shown in our previous simulations, and also to the relatively low collision energies of the experiments.

6. KMC simulations predict the appearance of other products, although with low yields and at relatively high internal energies of the ion: acetylene and the $\mathrm{m} / \mathrm{z}$ 87 ion, molecular hydrogen and the $\mathrm{m} / z 111$ ion, and hydrogen isocyanide and the $\mathrm{m} / \mathrm{z} 86$ ion. These peaks are absent in the present experimental mass spectrum and their weighted theoretical yields are negligible, which is explained by their low (theoretical) yields and the high internal energies of the ion.

7. The algorithm employed in the present study has been designed to locate saddle points, and it misses dissociation channels proceeding without a saddle point, which might be important in the kinetics as they are entropically favored. For this reason, in the future it would be of interest to automatically find these dissociation pathways using multireference calculations

The present statistical study complements previous direct dynamics simulations on the same system. The combination of direct dynamics simulations to study short time-scales, alongside with the present automated procedure to map the PES and KMC/RRKM simulations to study the ion's fate in longer timescales, provides a useful tool to understand fragmentation mechanisms of biological molecules and to complement MS experiments.

\section{Acknowledgements}

This work was supported by Xunta de Galicia through project GRC2014/032 and ANR DynBioReact (Grant number ANR-14CE06-0029-01). The authors thank "Centro de Supercomputación de Galicia (CESGA)" for the use of their computational facilities.

\section{Notes and references}

${ }^{a}$ Université d'Evry Val d'Essonne, Laboratoire Analyse et Modélisation pour la Biologie et l'Environnement, Boulevard François Mitterrand,

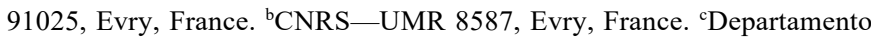
de Química Física and Centro Singular de Investigación en Química Biológica y Materiales Moleculares (CIQUS), Campus Vida, Universidade de Santiago de Compostela, 15782, Santiago de Compostela, Spain. Email: riccardo.spezia@univ-evry.fr and emilio.nunez@usc.es

Electronic Supplementary Information (ESI) available: Computational details of the TSSCDS method, electronic energies, optimized geometries and vibrational frequencies of the stationary points of the system, and details of the kinetic calculations. See DOI: 10.1039/b000000x/

1. E. Martínez-Núñez, Phys. Chem. Chem. Phys., 2015, 17, 14912

2. E. Martínez-Núñez, J. Comput. Chem., 2015, 36, 222.

3. S. A. Vazquez and E. Martinez-Nunez, Phys. Chem. Chem. Phys. $2015,17,6948$.

4. Z. Homayoon, S. A. Vázquez, R. Rodríguez-Fernández and E. Martínez-Núñez, J. Phys. Chem. A, 2011, 115, 979.

5. M.-P. Gaigeot and R. Spezia, Top. Curr. Chem., 2015, 364, 99.

6. P. DeSainteClaire and W. L. Hase, J. Phys. Chem., 1996, 100, 8190 .
O. Meroueh and W. L. Hase, J. Phys. Chem. A, 1999, 103, 3981. R. Spezia, J.-Y. Salpin, M.-P. Gaigeot, W. L. Hase and K. Song, J. Phys. Chem. A, 2009, 113, 13853.

9. E. Martínez-Núñez, S. A. Vázquez and J. M. C. Marques, J. Chem Phys., 2004, 121, 2571

10. E. Martinez-Nunez, S. A. Vazquez, F. J. Aoiz and J. F. Castillo, $J$ Phys. Chem. A, 2006, 110, 1225.

11. E. Martínez-Núñez, A. Fernández-Ramos, S. A. Vázquez, J. M. C. Marques, M. Xue and W. L. Hase, J. Chem. Phys., 2005, 123, 154311.

12. R. Rodriguez-Fernandez, S. A. Vazquez and E. Martinez-Nunez, Phys. Chem. Chem. Phys., 2013, 15, 7628.

13. D. Ortiz, P. Martin-Gago, A. Riera, K. Song, J.-Y. Salpin and R. Spezia, Int. J. Mass. Spectrom., 2013, 335, 33

14. R. Spezia, J. Martens, J. Oomens and K. Song, Int. J. Mass. Spectrom., 2015, 388, 40 .

15. R. Spezia, S. B. Lee, A. Cho and K. Song, Int. J. Mass. Spectrom., 2015, 392, 125 .

16. D. Ortiz, J.-Y. Salpin, K. Song and R. Spezia, Int. J. Mass. Spectrom., 2014, 358, 25.

$17 . \quad$ Y. Jeanvoine, M.-P. Gaigeot, W. L. Hase, K. Song and R. Spezia, Int. J. Mass Spectrom., 2011, 308, 289.

18. A. Martín-Sómer, M. Yáñez, W. L. Hase, M.-P. Gaigeot and R. Spezia, J. Chem. Theor. Comput., 2016, 12, 974.

19. R. Spezia, A. Cimas, M.-P. Gaigeot, J.-Y. Salpin, K. Song and W. L. Hase, Phys. Chem. Chem. Phys., 2012, 14, 11724.

20. A. Martin-Somer, M. Yanez, M.-P. Gaigeot and R. Spezia, J. Phys. Chem. A, 2014, 118, 10882.

21. E. Rossich-Molina, D. Ortiz, J.-Y. Salpin and R. Spezia, J. Mass. Spectrom., 2015, 50, 1340.

22. J.-Y. Salpin, S. Guillaumont, J. Tortajada, L. MacAleese, J. Lemaire and P. Maitre, ChemPhysChem, 2007, 8, 2235.

J. J. P. Stewart, J. Mol. Model., 2013, 19, 1.

K. Fukui, Acc. Chem. Res., 1981, 14, 363.

J. J. P. Stewart, MOPAC2012, Stewart Computational Chemistry. , Colorado Springs, CO, USA. Available at: http://OpenMOPAC.net.

26. M. J. Frisch, G. W. Trucks, H. B. Schlegel, G. E. Scuseria, M. A. Robb, J. R. Cheeseman, G. Scalmani, V. Barone, B. Mennucci and G. A. Petersson, et. al. Gaussian 09 (2009) Gaussian Inc., Wallingford CT.

F. Pietrucci and W. Andreoni, Phys. Rev. Lett., 2011, 107, 085504. B. Bollobas, Modern Graph Theory, Springer-Verlag, Berlin, 1998. G. Smith and R. G. Gilbert, Theory of unimolecular and recombination reactions, Blackwell Scientific Publications, Oxford, 1990.

30. A. B. Bortz, M. H. Kalos and J. L. Lebowitz, J. Comput. Phys., $1975,17,10$

D. T. Gillespie, J. Comput. Phys., 1976, 22, 403.

32. O. Beruski and L. N. Vidal, J. Comput. Chem., 2014, 35, 290.

32. O. Beruski and L. N. Vidal, J. Comput. Chem., 2014, 35, 290.
33. G. H. Peslherbe and W. L. Hase, J. Chem. Phys., 1994, 101, 8535.

34. J. J. P. Stewart, J. Comput. Chem., 1989, 10, 209.

35. S. Chapman and D. L. Bunker, J. Chem. Phys., 1975, 62, 2890.

36. C. S. Sloane and W. L. Hase, J. Chem. Phys., 1977, 66, 1523.

37. W. L. Hase, R. J. Duchovic, X. Hu, A. Komornicki, K. F. Lim, D.H. Lu, G. H. Peslherbe, K. N. Swamy, S. R. Vande Linde, A. J. C. Varandas, H. Wang and R. J. Wolf, VENUS96, a general chemical dynamics computer program, (1996).

38. J. J. P. Stewart, J. Comput. Aided Mol. Des., 1990, 4, 1.

39. J. Liu, B. W. Uselman, J. M. Boyle and S. L. Anderson, J. Chem. Phys., 2006, 125, 133115.

40. I. V. Chernushevich, B. A. Loboda and B. A. Thomson, J. Mass. Spectrom., 2001, 36, 849 .

41. M. Monge-Palacios, J. J. Nogueira and E. Martinez-Nunez, J. Phys. Chem. C, 2012, 116, 25454.

42. J. J. Nogueira, W. L. Hase and E. Martínez-Núñez, J. Phys. Chem. $C, 2014,118,2609$

43. P. DeSainteClaire, G. H. Peslherbe and W. L. Hase, J. Phys. Chem., $1995,99,8147$.

44. T. Baer and W. L. Hase, Unimolecular Reaction Dynamics, Oxford, 1996.

C. C. Nelson and J. A. McCloskey, J. Am. Chem. Soc. Mass. Spectrom., 1994, 5, 339. 
46. D. G. Beach and W. Gabryelski, J. Am. Chem. Soc. Mass. Spectrom., 2012, 23, 858 .

47. L. Sadr-Arani, P. Mignon, H. Chermette and T. Douki, Chem. Phys. Lett., 2014, 605-606, 108. 